\title{
Prevención del Fracaso Escolar en Educación Secundaria en Andalucía desde la Voz del Profesorado. Estudio de Caso
}

\author{
Prevention of School Failure in Comprehensive School in \\ Andalusia from the Voice of Teachers. A Case Study
}

\author{
Prevenção da Evasão Escolar do Ensino Secundário na \\ Andaluzia já que a Voz dos Professores. Estudo de Caso
}

Jesús Domingo * José M. Martos

Universidad de Granada

\begin{abstract}
El fracaso escolar es un concepto complejo. Suele achacarse a los sujetos como porcentualmente inevitable y se ignoran los demás fracasos convergentes (metodológicos, curriculares, organizativos...). Los centros poseen autonomía pedagógica para afrontar este reto. Este estudio de caso presenta una medida de apoyo en contraste con la realidad del aula ordinaria. Utiliza relatos de experiencia y entrevistas en profundidad. Destaca la importancia de prevenir la construcción de trayectorias consumadas de fracaso escolar. Reivindica la flexibilización curricular, la personalización de los procesos de enseñanza-aprendizaje, mediante metodologías activas y participativas y las altas expectativas sobre el alumnado.
\end{abstract}

Descriptores: Fracaso escolar, Organización, Competencias para la vida, Clase.

School failure is a complex concept. It is usually attributed to the subjects as percentage inevitable and the other convergent failures are ignored (methodological, curricular, organizational ...). Schools have pedagogical autonomy to face this challenge. This case study presents a measure of support in contrast to the reality of the ordinary classroom. We use stories of experience and in-depth interviews. It emphasizes the importance of preventing the construction of consummate trajectories of school failure. The article calls for curricular flexibility, personalization of teaching-learning processes, through active and participative methodologies and high expectations about students.

Keywords: Academic failure, Organization, Life skills, School classes.

O fracasso escolar é um conceito complexo. Geralmente atribuída aos sujeitos como uma percentagem inevitável e outros convergiram falhas (metodológicos, curriculares, organizacional ...) são ignoradas. Os centros têm autonomia pedagógica para enfrentar este desafio. Este estudo de caso apresenta uma medida de apoio em contraste com a realidade da sala de aula comum. Use relatos de experiência e entrevistas. Ele salienta a importância de prevenir a construção de fracasso escolar caminhos consumados. Vindica a flexibilidade curricular, personalização do ensino-aprendizagem através de metodologias ativas e participativas e grandes expectativas para os alunos.

Palavras-chave: Fracasso escolar, Organização, Habilidades para a vida, Sala de aula.

* Contacto: jdomingo@ugr.es

ISSN: 2254-3139

www.rinace.net/riejs/

revistas.uam.es/riejs
Recibido: $\quad 7$ de enero 2017

$1^{a}$ Evaluación: 13 de febreo 2017

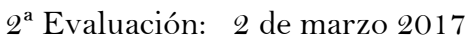

Aceptado: $\quad 16$ de marzo 2017 
El estudio forma parte de una línea de investigación del Grupo FORCE de la Universidad de Granada (HUM-386) y de la "Red de Investigación sobre Liderazgo y Mejora Educativa" (RILME), sobre mejora escolar en contextos desafiantes. Surge a partir del proyecto de Investigación I+D+i titulado "Estudiantes en riesgo de exclusión educativa en la ESO: Situación, programas y buenas prácticas en la Comunidad Autónoma de Andalucía" (Ref. SEJ2006-14992-CO6-O4), con continuidad en el actual sobre "Liderazgo pedagógico y desarrollo del centro como comunidad profesional: prácticas de éxito en educación obligatoria” (Ref. EDU2013-48432-P).

\section{Presentación y justificación del problema de investigación y su abordaje teórico}

El fracaso escolar y la exclusión educativa son conceptos multidimensionales y complejos (Escudero, 2005, 2013; Escudero, González González y Martínez Domínguez, 2009; Jiménez, Luengo y Taberner, 2009). Ambos, con la concurrencia de una combinación compleja de variables intervinientes y una acumulación de obstáculos que terminan por afectar al alumnado a lo largo de su vida, son resultado de una escolarización que no garantiza el éxito para todos. En cambio, se suelen atribuir y achacar al sujeto que lo sufre, sin reparar en los procesos de violencia estructural que viven $\mathrm{ni}$ en el resto de fracasos curriculares, profesionales, metodológicos u organizativos que coadyuvan a consolidar estas situaciones y trayectorias.

Las cifras y estadísticas oficiales [...] son contundentes al respecto, pero también resultan abstractas y frías. Llegan a documentar que el fracaso tiene color de clase social, de minorías, etnia, sexo o capital cultural de las familias, pero la lógica de los grandes números tiende a dejar en la sombra por qué y cómo ocurren las cosas de ese modo. (Escudero, González González y Martínez Domínguez, 2009, p. 45)

Son varios los autores (Fernández, Mena y Reviere, 2010; Karsz, 2004; Tarabini, 2015) que denuncian esta situación y los silencios, olvidos y normalización de las trayectorias de bajo rendimiento, que suponen claramente un riesgo de desapego y exclusión (Canario, 2000). Especialmente en poblaciones y contextos en los que se asume, desde una cierta ética utilitarista, la situación como "responsabilidad de los sujetos" y "porcentualmente" normal, quedando en no pocos casos invisibilizada (Escudero, 2005; Escudero y Rodríguez, 2016, p. 83) por no provocar situaciones especialmente disruptivas al conjunto o por considerar el profesorado que sus causas están fuera de su control (Tarabini, 2015).

Voces autorizadas (Escudero, González González y Martínez Domínguez, 2009; Tarabini, Curran, Montes y Parcerisa, 2015) recomiendan que más que hablar de fracaso escolar como producto final, interesa considerar esta realidad desde la perspectiva de la exclusión social, y abordarlo de forma dinámica, como un proceso, una trayectoria de vulnerabilidad educativa e inclusión incompleta que puede desencadenar exclusión educativa (más o menos encubierta) y, finalmente, fracaso escolar (como no titulación). Por lo que proponen actuar sobre los diferentes factores disruptores (muchos de ellos estructurales y escolares) y los aprendizajes esenciales que garantizan una buena educación y el éxito escolar. Lo que deja margen a la acción, a la reconstrucción de los procesos educativos desde una perspectiva de prevención o de prácticas transformadoras, actuando tempranamente y reconduciendo trayectorias personales y académicas de bajo rendimiento con medidas específicas y proyectos educativos comunitarios verdaderamente inclusivos que caminen hacia el éxito para todos y entre todos y 
minimice riesgo de consumación del fracaso escolar o el distanciamiento con la educación.

Para dar respuesta a los nuevos retos planteados, en el seno de los centros educativos y bajo la denominación genérica de "atención a la diversidad", han sido múltiples los cambios organizativos, metodológicos y didácticos (Ainscow, Booth y Dyson, 2006; Rodríguez, 2013; Sevilla, 2003). No obstante, el conocimiento acumulado informa recurrentemente que la escuela actual, lejos de asegurar la justicia y el éxito para todos, reproduce, genera o asume la inevitabilidad del fracaso escolar (Bolívar, 2005).

Las medidas que suponían adaptaciones, currículum paralelos y medidas organizativas diferentes... significan de hecho un doble currículum para este alumnado (el de clase y el de apoyo), que genera desconcierto, apoyo-dependencia y un paulatino incremento de la distancia (afectiva, relacional, cognitiva y de resultados de aprendizaje) entre ellos y el resto del grupo clase. Si bien es verdad que el alumnado que participa en medidas de apoyo, cuando se hace de manera eficiente, cambia su actitud hacia el aprendizaje, con posibilidades de reinserción y éxito (Aramendi, Vega y Santiago, 2011; Luzón, Porto, Torres y Ritacco, 2009; Vega y Aramendi, 2009), si no se vence el apego a estas medidas de apoyo y se rechaza al aula ordinaria, no se ha conseguido la verdadera inclusión.

Numerosas investigaciones ponen de manifiesto que dichas medidas, por sí mismas y tal y como están siendo implementadas, una vez alcanzados los niveles de escolarización para todos, no sólo no están consiguiendo los resultados deseados, sino que en muchas ocasiones están contribuyendo a crear un nuevo proceso de dualización de la enseñanza (Echeita, et al., 2009; Fernández, Mena y Reviere, 2010; Martínez, 2010). Por ello, no sin razón, Correia (2004) denuncia la injusticia y paradoja del sistema, porque muchas de las medidas pensadas e implementadas para la inclusión, en verdad contribuyen a la exclusión, llamándolas así "medidas de inclusión para la exclusión”.

Prevenir la consolidación de las trayectorias de fracaso y exclusión educativa requiere una educación pública de calidad en la que se entrecruzan los principios y procesos de desarrollo de la escuela inclusiva (Ainscow, Booth y Dyson, 2006) y la consideración del derecho a un buen aprendizaje para todos (Armstrong y otros, 2009; Darling-Hamond, 2001; UNESCO, 2008), incrementando el éxito (OCDE, 2016). Es decir, avanzar en la controvertida idea de compaginar calidad y equidad que propone el replanteado movimiento de School/educational Effectiveness Research (SER) (Murillo, 2004; Murillo y Hernández-Castilla, 2011; Sammons, 2007). Lo que se hace especialmente complejo en educación secundaria.

Compatibilizar la equidad y el derecho a una buena educación y un buen aprendizaje para todos requiere abordajes alternativos (OCDE, OIE-UNESCO, UNICEF LACRO, 2016; Sammons y Bakkum, 2011; Valls, Prados y Aguilera, 2014), tanto en el aula de referencia como en cualquier tipo de medida de apoyo. Si las trayectorias escolares pueden variar drásticamente hacia el aprendizaje o hacia la paulatina consolidación de trayectorias de exclusión y terminar en fracaso, importa tomar en consideración qué puede estar ocurriendo en las aulas de secundaria, desde voces autorizadas, para repensarlas e intervenir al respecto.

En este sentido interesa de un lado conocer buenas prácticas de atención a la diversidad y de respuesta a estas situaciones de vulnerabilidad escolar, como antídotos a itinerarios consolidados de no éxito escolar; y, de otro, más específicamente, conocer experiencias 
de centros educativos que obtienen unos resultados aceptables y que, además, toman medidas preventivas específicas frente a la exclusión educativa. El estudio sobre estas realidades puede ser una llamada de atención al resto de la escuela por si se está sobrecargando el currículum, adornándolo o dejando de lado lo esencial. De este modo, lo que se aprende de estudiar buenas prácticas de medidas de atención a la diversidad puede ayudar también al resto de la escuela.

Conscientes de que el tema reclama un acercamiento holístico y situado para alcanzar una comprensión que ilumine las dinámicas que subyacen a las mismas, se presta especial atención a los discursos que se generan para describir y explicar estos procesos. Se repara, en este sentido, en el del profesorado que trabaja en las medidas objeto de estudio. Este discurso ayuda a comprender su visión sobre las trayectorias de fracaso del alumnado y los planteamientos didáctico-pedagógicos que ponen en marcha frente a ello.

Estos profesionales, como responsables y testigos de las medidas de atención y apoyo educativo, son a la luz de la investigación actual (Clandinin y Connelly, 2000; Susinos y Parrilla, 2008; Tarabini, 2015) informantes clave. La voz del profesorado nos permite entrar a comprender desde dentro esta compleja realidad. El diálogo en profundidad con ellos, permite un acercamiento holístico y situado, capaz de poner en debate su conocimiento práctico profesional, iluminando expectativas, percepciones y otras dinámicas que subyacen a las mismas, y que sustentan sus principios profesionales sobre atención a la diversidad.

\section{Objetivo de investigación}

Caracterizar y comprender las concepciones implícitas, orientaciones e intencionalidades que subyacen en el discurso del profesorado que participa en medidas preventivas del fracaso escolar en educación secundaria.

\section{Metodología}

Se busca identificar claves de prevención de la consolidación de trayectorias de fracaso desde la voz del profesorado que participa en una medida preventiva de la consolidación de trayectorias de fracaso escolar en un instituto de educación secundaria en Andalucía. Para ello se realizó un estudio de caso.

Se utiliza una metodología comunicativa (Gómez, Latorre, Sánchez Aroca, y Arrow, 2006), para superar la brecha epistemológica entre investigadores y profesorado. Se aborda desde la voz y relatos de experiencia del profesorado que participa en ella. Estos profesionales, que narran su historia de apoyo, son los mejores conocedores, responsables y testigos de qué ocurre en las aulas y qué impacto tiene sobre su alumnado. Sus relatos de experiencia profesional transmiten su conocimiento práctico, lo que tiene gran valía formativa y para la mejora (Clandinin y Connelly, 2000).

\subsection{Contexto e informantes clave}

Se buscó intencionalmente un instituto de educación secundaria con un índice socioeconómico y cultural medio, con una gran diversidad cultural, que se define y se le reconoce como inclusivo y que obtiene unos resultados académicos adecuados a su 
contexto. Para confrontar y precisar dicha selección se revisaron los resultados escolares y educativos del curso escolar 2013/2014.

El caso seleccionado es el Instituto de Educación Secundaria "El Palmeral" de Vera (Almería). Este centro, en función de su autonomía pedagógica, entre otras medidas de atención a la diversidad (que no son objeto de este estudio), desarrolla una propuesta en la primera etapa de la ESO, que trabaja en grupos flexibles en función de su competencia curricular. Se trata de una medida diseñada y desarrollada en el marco de autonomía pedagógica de los centros educativos españoles y al amparo de las orientaciones de la Orden de 25 de julio de 2008 en Andalucía, que otorga a los centros educativos de potestad para promover, diseñar y desarrollar medidas, programas y adecuaciones organizativas y curriculares como respuesta a los desafíos educativos de la diversidad y los de atender adecuadamente a todos, en un marco inclusivo. Estos grupos se gradúan en función de las necesidades del alumnado. En particular nos detenemos en una medida que ellos denominan "prediversificación". El objetivo de la misma es ofertar una experiencia de aprendizaje más cercana a las necesidades y ritmos del alumnado en incipiente riesgo de exclusión educativa.

No se trata tanto de analizar la medida en sí, cuanto de comprender las razones y principios pedagógicos del profesorado que participa en ella de cara a inferir aprendizajes para la prevención del fracaso escolar en secundaria a partir de esta experiencia particular.

Los informantes clave seleccionados son productores de información primaria en dialéctica con el investigador. Son seleccionados de manera intencional en función de su dilatada experiencia profesional en el ámbito y su permanencia en este tipo de acciones. Responden a las variables recogidas en la tabla 1.

Tabla 1. Informantes clave

\begin{tabular}{lcccc}
\hline \multicolumn{1}{c}{ ATRIBUTOS } & INFORMANTE 1 & INFORMANTE 2 & INFORMANTE 3 & INFORMANTE 4 \\
\hline Género & Masculino & Femenino & Masculino & Masculino \\
\hline $\begin{array}{c}\text { Experiencia } \\
\text { profesional }\end{array}$ & 19-30 años & $7-17$ años & 19-30 años & 19-30 años \\
\hline $\begin{array}{c}\text { Participa equipo } \\
\text { directivo }\end{array}$ & Sí No & Sí & No \\
\hline $\begin{array}{c}\text { Trayectoria } \\
\text { profesional }\end{array}$ & $\begin{array}{c}\text { ESO, Bachillerato, } \\
\text { FP }\end{array}$ & ESO Bachillerato & Primaria ESO & $\begin{array}{c}\text { ESO } \\
\text { Bachillerato }\end{array}$ \\
\hline Titulación & Licenciatura & Licenciatura & Magisterio & Licenciatura \\
\hline
\end{tabular}

Fuente: Elaboración propia.

\subsection{Procedimiento}

Se utilizan los relatos de experiencia práctica de este profesorado y las entrevistas dialógicas en profundidad con los mismos. Tras un proceso de preguntas constantes y con una codificación emergente de los relatos (Birks y Mills, 2015), se profundizan desde el análisis crítico del discurso (Van Dijk, 2012). Estas metodologías ayudan a caracterizar los discursos y, mediante abordajes diferentes y preguntas constantes, llegar a los principios pedagógicos que sustentan la práctica profesional y la toma de decisiones sobre el aprendizaje de estos estudiantes. Con ello se muestran, ubican y reconceptualizan los discursos del profesorado sobre sus experiencias profesionales de atención a la diversidad cuando existen historias de fracaso escolar y riesgo de abandono 
temprano. Esta comprensión, aún en su particularidad y especificidad, permite obtener aprendizajes útiles a tomar en consideración a la hora de desarrollar modelos didácticos de educación para todos y entre todos, aplicables en el aula ordinaria.

A partir de los relatos, y por medio de un proceso de codificación abierta, se establecen unas categorías emergentes del análisis que se han agrupado en subdimensiones y dimensiones que se integran, a su vez, en los tres bloques de significado teórico: caracterización de la medida, del alumnado y de la clase-aula (tabla 2).

Tabla 2. Sistema de categorías utilizado

\begin{tabular}{|c|c|c|c|}
\hline BLOQUES & $\begin{array}{l}\text { DIMENSIONES DE } \\
\text { ANÁLISIS }\end{array}$ & SUB-DIMENSIONES DE ANÁLISIS & $\begin{array}{l}\text { REFERENCIAS } \\
\text { CODIFICADAS }\end{array}$ \\
\hline Descripción c & a medida & & 15 \\
\hline \multirow{4}{*}{ Alumnado } & Perfil y trayectoria & & 10 \\
\hline & \multirow{3}{*}{ Problemáticas } & $\begin{array}{l}\text { Factores vinculados al ámbito } \\
\text { personal }\end{array}$ & 18 \\
\hline & & $\begin{array}{l}\text { Factores vinculados al ámbito } \\
\text { familiar }\end{array}$ & 12 \\
\hline & & $\begin{array}{l}\text { Factores vinculados al ámbito } \\
\text { educativo }\end{array}$ & 20 \\
\hline \multirow{4}{*}{ Clase-aula } & \multirow{2}{*}{ Clase-aula de referencia } & Enseñanza-aprendizaje & 5 \\
\hline & & Experiencia del alumnado & 13 \\
\hline & \multirow{2}{*}{$\begin{array}{l}\text { Clase-aula de } \\
\text { prediversificación }\end{array}$} & Enseñanza-aprendizaje & 62 \\
\hline & & Experiencia del alumnado & 25 \\
\hline Total & & & 180 \\
\hline
\end{tabular}

Fuente: Elaboración propia.

Paralelamente, y con la intencionalidad de buscar las inferencias y relaciones que encierran los discursos del profesorado, necesarias para su comprensión en profundidad, se ha realizado un proceso de codificación axial. Los resultados de ambos procesos de codificación se presentan integradamente en mapas conceptuales.

Se utilizan en estos procesos de análisis y codificación las herramientas del programa Nvivo 1.

Se toman en consideración de forma escrupulosa los criterios éticos de investigación y, atentos a que la investigación sea además verdaderamente inclusiva (Parrilla, 2009), más que volver sobre los tópicos de las dificultades y la caracterización de una medida particular -y por ello difícilmente replicable-, se levantan cuestiones a debate a partir de los discursos emergentes en una experiencia que actúa preventivamente frente a la exclusión educativa.

\section{Resultados}

Los resultados de investigación se concentran en tres grandes bloques de contenido que se describían con anterioridad en el tabla 2: 1) descripción de la medida; 2) factores vinculados a las trayectorias de fracaso del alumnado; y 3) factores vinculados con el tipo de agrupamiento, con sus respectivas subdimensiones. Cada bloque y dimensión se comenta seguidamente en diferentes apartados. 


\subsection{Descripción de la medida}

El profesorado ofrece una descripción de lo que ellos denominan "prediversificación". Lo específico de ella, frente al funcionamiento normal del aula de referencia, radica en trabajar en grupos reducidos las materias instrumentales, integrando los contenidos con un solo profesor por ámbito de trabajo. Para el resto de asignaturas vuelven a su aula de referencia. Son grupos de chicos y chicas con incipientes trayectorias de fracaso escolar, vulnerables a sufrir procesos de exclusión educativa, y que a juicio del equipo docente tienen posibilidades de éxito si se son atendidos de manera más personalizada.

Hemos sacado el alumnado de los dos grupos de primero que tienen algunas dificultades de aprendizaje y no participan en la medida de apoyo a la integración. Se ha creado un grupo de prediversificación en el que trabajamos por ámbitos. Yo trabajo con ellos una programación integrada de matemáticas y ciencias naturales. (Inf1Ref2)

La medida se inscribe dentro de la trayectoria de atención a la diversidad del centro. El trabajo previo en grupos flexibles y la experiencia en la diversificación curricular confluyó en la articulación de esta propuesta de trabajo.

En 2007 se crearon los grupos flexibles. Habia dos grupos, uno a nivel alto y otro bajo, para atender mejor a la diversidad. Para no hacer compartimentos cerrados se estimulaba la movilidad del alumnado. Esto constituyó un gran paso. (Inf2Ref2)

De ahí nacen estos grupos como una medida preventiva de atención a la diversidad que no comporta una adaptación curricular significativa ni cataloga al alumnado. Lo específico es la metodología y la interacción con el alumnado que permite establecer unas dinámicas flexibles e individualizadas distintas a las que impregnan el proceso de enseñanza-aprendizaje en un grupo numeroso y academicista.

\footnotetext{
En este grupo se siguen manteniendo los objetivos educativos del grupo de referencia, sólo que trabajan a otro ritmo, dedicando más horas a los aspectos que así lo requieran. Por ejemplo, tengo siete horas de clase y esto me permite dedicar más tiempo a una asignatura o a otra, según conviene en cada momento. (Inf3Ref7)
}

\subsection{El alumnado}

El profesorado señala como condición para participar en esta medida: tener interés, a pesar de las dificultades que pueda arrastrar a nivel educativo e indistintamente de los "dictámenes de escolarización" (Inf1Ref15; Inf2Ref9; Inf4Ref4). "Se trata de un alumnado que siguiendo el proceso normal de la ESO no funciona, va a fracasar y en cambio, de este modo puede obtener buenos resultados" (Inf1Ref15).

La oferta formativa del instituto también determina el perfil del alumnado. Al ser un centro bilingüe, participan en esta medida alumnado que en el primer curso de la ESO estaban en grupos bilingües, buscando una mayor calidad de la enseñanza, pero que no alcanzaron los objetivos deseados para esta modalidad educativa, iniciando un proceso de fracaso escolar. (Inf4Ref5)

Por último, también participan otros que experimentan problemáticas vinculadas a la discapacidad o a la desventaja socioeducativa en razón de la etnia; aunque no es el grupo más numeroso. (Inf4Ref5)

En sus discursos el profesorado se detiene en señalar las problemáticas que rodean las trayectorias de este alumnado en el que confluyen diversos factores vinculados al ámbito personal, familiar y educativo. 


\section{a) Factores vinculados al ámbito personal}

El profesorado describe las principales problemáticas que indicen en sus trayectorias educativas desde el ámbito personal: los "problemas superables de motivación" (Inf1Ref29; Inf3Ref10,20) y "la falta de hábito de trabajo” (Inf1Ref10,4; Inf3Ref2 1) que tiene como consecuencia la "dificultad para operativizar sus capacidades" (Inf3Ref20).

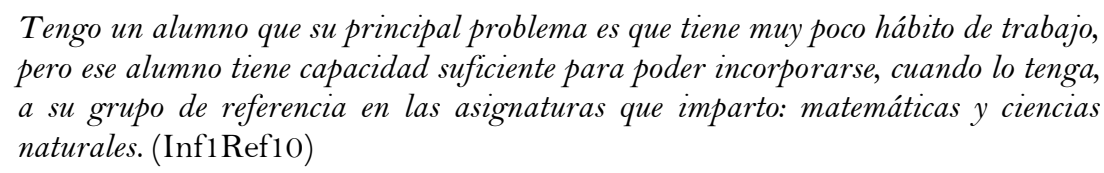

En otros casos es la facilidad para distraerse y "perderse en el gran grupo" (Inf1Ref15) o la necesidad de un estímulo inmediato para atender (In4Ref26; Inf1Ref18). Esto hace que se hayan ido desenganchando progresivamente en su trayectoria educativa. Por último, “existe un conjunto menos significativo de otras problemáticas” (Inf35Ref20; Inf1Ref10).

\section{b) Factores vinculados al ámbito familiar}

El grado de apoyo e interés de las familias de este alumnado es diverso (Inf1; Inf4), lo que "genera distintos grados de implicación y colaboración en el trabajo de los hijos en casa” (Inf1Ref10). De todos estos comentarios resulta relevante señalar que, a juicio del profesorado, muchos de estos padres no aceptan que sus hijos participen en la medida. No entienden que se trata de una medida preventiva y transitoria.

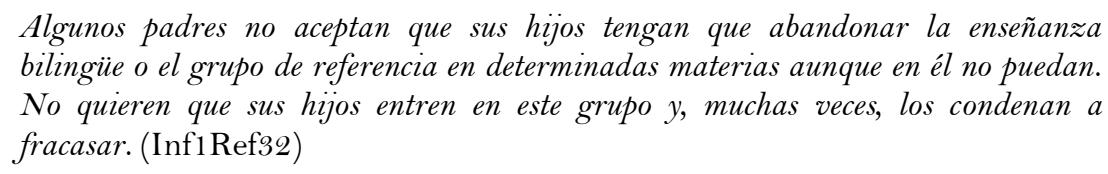

\section{c) Factores vinculados al ámbito educativo}

Las anteriores problemáticas, vinculadas al ámbito personal y familiar, confluyen en el ámbito escolar configurando "una trayectoria educativa marcada por las dificultades para aprender” (Inf1Ref18; Inf3Ref20) y alcanzar "los objetivos establecidos en el currículum” (Inf1Ref15), llegando incluso a "estar acostumbrados a suspender cualquier prueba de evaluación” (Inf3Ref11).

El profesorado señala algunos factores que abocan al alumnado a una situación de riesgo: los "déficit en lectoescritura que les dificulta la comprensión”(Inf1Ref18) y "la realización de las tareas" (Inf3Ref24); "la falta de autonomía en el trabajo de aula y en el de casa" (Inf3Ref10.11) y "la necesidad de reforzar continuamente los aprendizajes adquiridos" (Inf3Ref10; Inf4Ref26).

Este conjunto de factores ha generado un retraso educativo acumulado y un desenganche progresivo o, en algunos casos, ha supuesto "el abandono del itinerario bilingüe” (Inf3Ref20). El no intervenir en el primer ciclo de educación secundaria significaría abocar al alumnado a un fracaso escolar consumado.

Hay alumnos que vienen con un retraso escolar acumulado, quizás por falta de motivación o por falta de una intervención adecuada en el momento oportuno. Esto puede ser perfectamente recuperable, podrán pasar a otro tipo de itinerario $y$ compartir el ritmo de aprendizaje de su grupo de referencia. (Inf3Ref2o) 


\subsection{La clase-aula}

El discurso del profesorado en torno a la clase-aula se construye dialógicamente contraponiendo las dinámicas y las experiencias vinculadas al aula de referencia frente a la de "prediversificación".

\subsubsection{La clase-aula de referencia}

Escrutando los términos sobre los que gira el discurso para caracterizar el aula de referencia, encontramos los verbos "atender", "funcionar", "participar" en forma negativa, para describir la respuesta a las necesidades educativas de los alumnos y las experiencias que se generan en esta aula.

El discurso del profesorado se articula en torno a dos bloques de categorías: enseñanzaaprendizaje y experiencia del alumno. Entre los descriptores de ambos se establece un conjunto de relaciones que se representan en el mapa conceptual (figura 1).

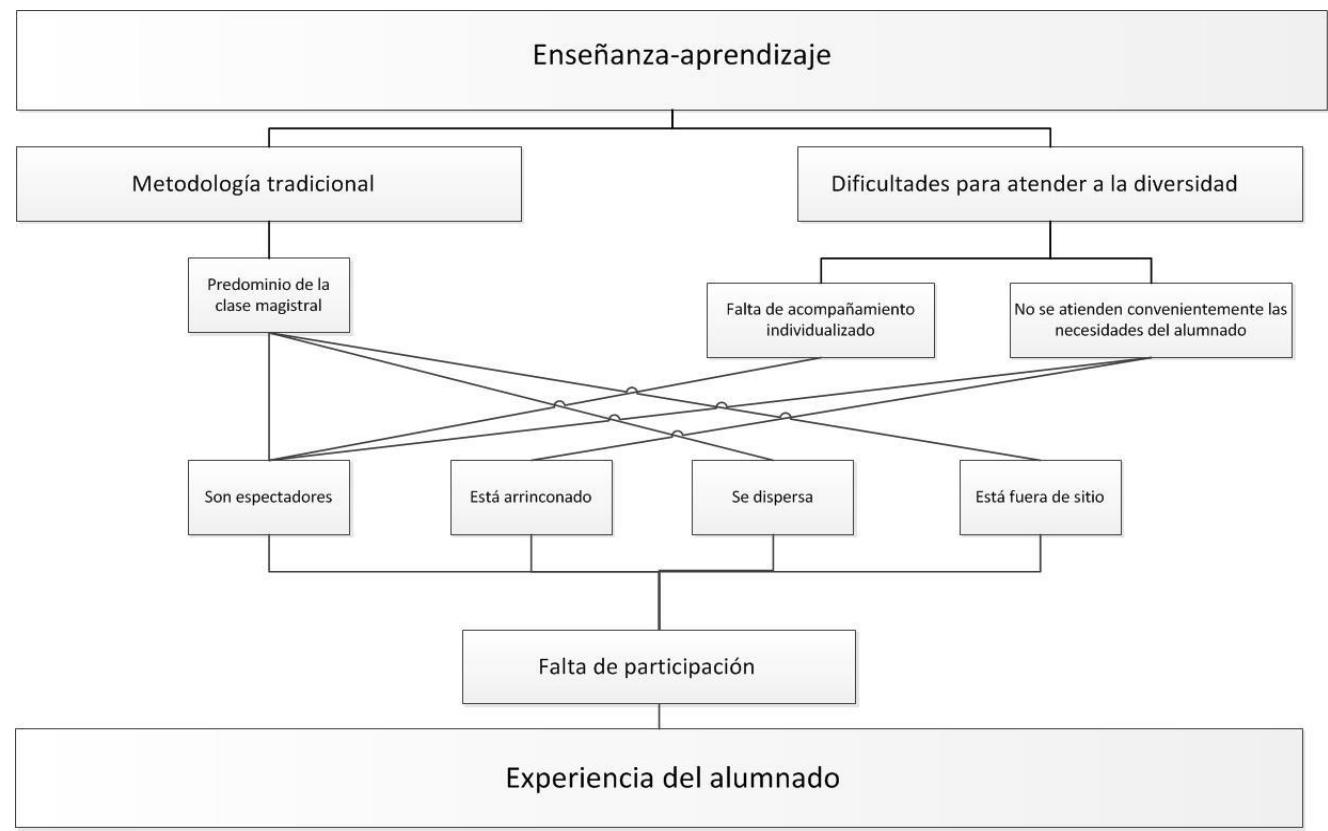

Figura 1. Interrelación de dimensiones en el discurso del profesorado sobre el aula de referencia

Fuente: Elaboración propia.

a) Enseñanza-aprendizaje

El profesorado vincula las problemáticas educativas que presenta este alumnado con la forma en la que se articulan los aprendizajes en el grupo de referencia. En "un aula numerosa, en la que metodológicamente predomina la clase magistral” (Inf4Ref26), al alumnado que tiene alguna dificultad, por leve que sea, no se le acompaña individualizadamente en su proceso de aprendizaje, ni se atiende convenientemente a sus necesidades. Esto genera una espiral de desventaja que conduce a que no trabajen ni aprendan en el aula:

Son alumnos que en el gran grupo no funcionan, porque no los puedes atender individualmente, no puedes atender a la diversidad. Estas mismas personas en el pequeño grupo funcionan muchísimo mejor, porque caminas junto a ellos. (Inf4Ref5) 
b) La experiencia del alumno

El no atender convenientemente a la diversidad del alumno va a determinar una vivencia y una experiencia de falta de participación en el aula, que el profesorado describe plásticamente con las siguientes imágenes: "es espectador" (Inf4Ref5), "no funcionan, están arrinconados, están perdidos, están fuera de sitio y se dispersan en la masa" $(\operatorname{Inf} 1 \operatorname{Ref15})$.

Ya no es que no quieran. Aunque quieran no pueden, porque ya están muy fuera de su sitio. $Y$ sobre todo, sin ningún hábito de trabajo. (Inf1Ref29)

\subsubsection{La clase-aula de prediversificación}

El profesorado habla sobre este agrupamiento de forma distinta del aula de referencia. En este caso su discurso se caracteriza por el uso en positivo de los verbos "trabajar", "atender", "reconocer", "motivar" para describir y caracterizar la experiencia de aprendizaje y el impacto que tiene en la trayectoria del alumnado.

La estructura de categorías resultante del análisis permite establecer dos grandes ámbitos temáticos con los que se caracteriza, una vez más, el proceso de enseñanzaaprendizaje y la experiencia del alumno en esta aula.

El análisis de conglomerados saca a la luz el entramado relacional que encierra el discurso del profesorado. En la figura siguiente se observa, de una parte, la idea de que conocer su progreso en la evaluación y la realización de las tareas es una fuente de motivación para el alumnado. De otra, conjuga los principios de flexibilización e individualización de procesos como fuente de la integración y participación del alumno en el proceso de enseñanza aprendizaje.

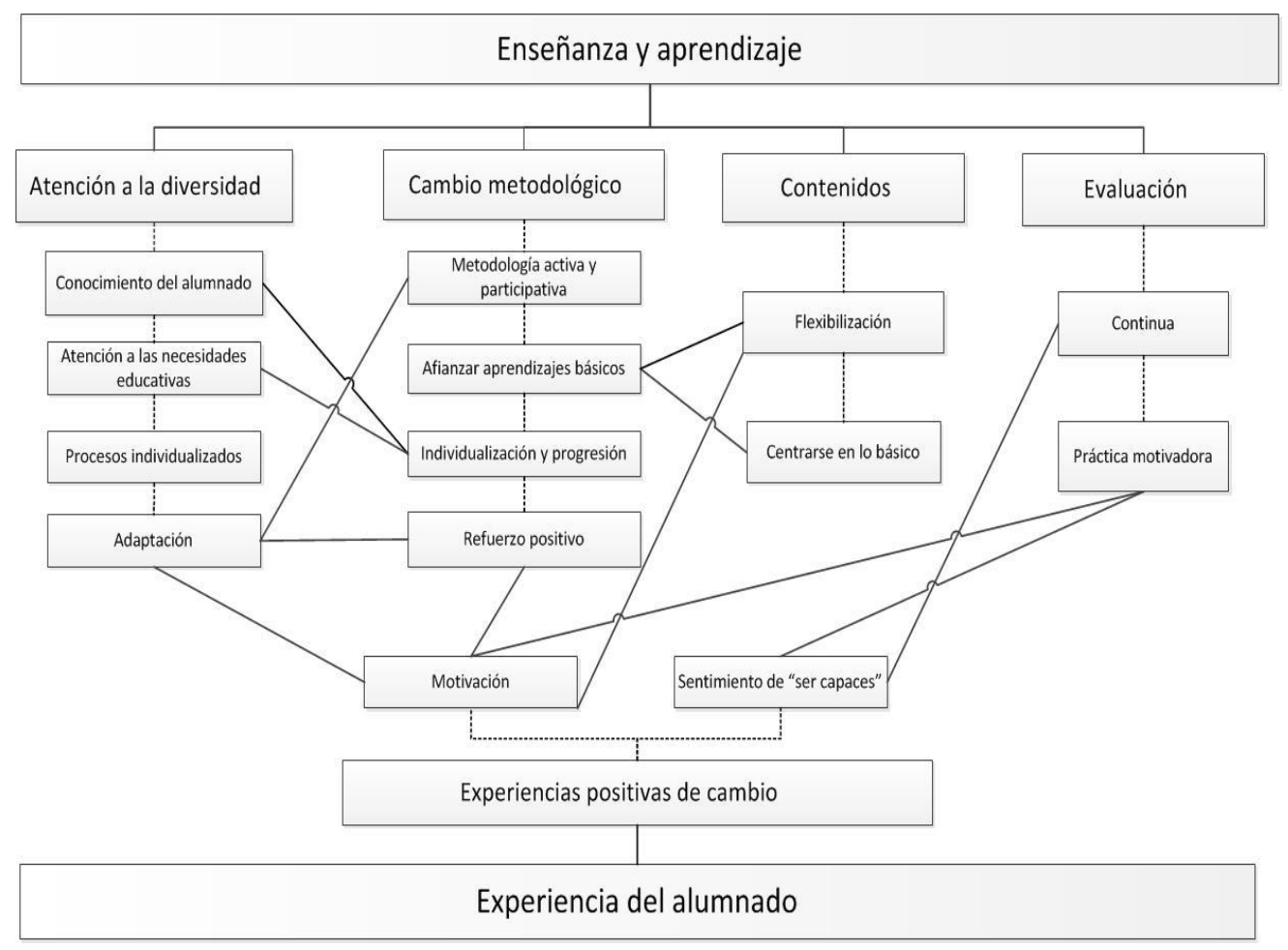

Figura 2. Interrelación de dimensiones en el discurso del profesorado sobre el aula de prediversificación

Fuente: Elaboración propia. 
a) Enseñanza-aprendizaje

\section{Atención a la diversidad}

La atención a la diversidad parte de tomar conciencia de la necesidad de conocer a cada alumno y alumna, adentrarse en su individualidad para adaptar el proceso de enseñanzaaprendizaje. En este sentido un profesor señala que "no es tanto el nivel lo que importa cuánto las personas a las que les estás dando clase” (Inf.4Ref4).

En el aula no todo el alumnado necesita la misma atención: puedes encontrarte personas con cierta discapacidad física, incluso personas cuyo problema principal es la exclusión social. Es un reto conocer a mis alumnos y una satisfacción a la vez. Como tienes tantas horas, les tomas muchísimo cariño. (Inf4Ref4)

Cuando la persona es el centro del proceso de enseñanza-aprendizaje, "el aula se convierte en un ámbito que alberga procesos individualizados" (Inf4Ref5) que se desarrollan a ritmos distintos. El profesorado considera que esta adaptación "sólo se puede realizar en un grupo pequeño, ya que permite dedicar más tiempo a cada alumno y alumna” (Inf1Ref10), "detectar cuándo se descuelgan de la dinámica de clase” (Inf4Ref35) y "dar el refuerzo positivo" (Inf1Ref27).

\section{Metodología}

Atender a la diversidad en el aula implica un cambio metodológico. "Más que una adaptación en contenidos es una adaptación en metodología” (Inf3Ref12). En efecto, es necesario señalar que el cambio en relación al aula de referencia no se produce a nivel de objetivos y contenidos sino a nivel metodológico, introduciendo nuevos modelos de trabajo, adaptando actividades y tareas a las necesidades y formas de aprender del alumnado.

Los contenidos en esta medida son los reglados. Cambiamos determinados ejercicios que tienen mayor grado abstracción y complicación para el alumnado. Trabajamos en el marco de las competencias básicas. (...) El alumnado marca el ritmo y, aunque parezca curioso, ahora mismo vamos avanzado más que en el grupo de referencia. Aqui la hora de clase la aprovechan, en el aula de referencia, como son más de veinte, el profesor no consigue avanzar, en algunos momentos, tanto como avanza en este grupo. (Inf1Ref1)

A la hora de seleccionar las actividades y tareas se procura priorizar aquellas que contribuyan a desarrollar y afianzar aprendizajes básicos para que sean capaces de transferirlos en otros contextos y a la resolución de problemas reales. (Inf1Ref9)

En Matemáticas tengo como objetivo que sepan leer e interpretar un problema sencillo. A ellos les cuesta mucho resolver problemas y los rechazan de entrada. Sólo les gusta realizar ejercicios de rutina. Por eso busco conseguir desarrollar una operatividad minima, pero sobre todo, que esa operación la sepan aplicar en un problema real (...) En Ciencias Naturales estoy centrándome en la lectura para propiciar la comprensión de la materia de Ciencias Naturales, pues si no te centras en la básico no puedes avanzar. (Inf1 Ref4)

Esta adaptación metodológica implica una progresión, ya que al afianzar aprendizajes básicos posibilitará enfrentarse a tareas de mayor envergadura en cada una de las asignaturas.

Es necesario trabajar, en un primer momento, con problemas sencillos para ir aumentando su dificultad. Pero, ante todo, no perder de vista lo básico. Son necesarias actividades de lectura y comprensión para extraer la información de los problemas y ver qué se les pide. Procurar que sean capaces de sacar los datos que tiene que utilizar e ir siguiendo un planteamiento lógico e identificar las etapas de 
un proceso. Son aprendizajes transferibles a otras materias y a otras situaciones. (Inf3Re35)

La progresión debe ir acompañada de una individualización del proceso, de forma que se respeten los ritmos de cada alumno para alcanzar unos objetivos comunes.

\begin{abstract}
Aunque se intente trabajar desde un nivel más o menos homogéneo, sin embargo, no puedes olvidar que unos pueden realizar diez ejercicios y otros, por su ritmo de trabajo, sólo la mitad. ¿Qué hacer? En este caso seleccionar el nivel de estos cinco ejercicios para que en ellos trabaje lo fundamental. (Inf3Ref2)
\end{abstract}

El profesorado describe un conjunto de pautas y orientaciones metodológicas que caracterizan su trabajo en el aula: realizar explicaciones breves "porque si no pierden la atención” (Inf3Ref27), “adaptarse a su ritmo de trabajo y graduar el nivel de dificultad de las tareas” (Inf3Ref28) y priorizar el trabajo en el aula frente a los deberes para la casa.

Con este alumnado hay que trabajar mucho para afianzar los aprendizajes. Hay que estar continuamente haciendo ejercicios en clase porque muchos de ellos en casa no trabajan. Pese a ello hay que procurar que trabajen en casa algo para conseguir desarrollar hábitos de trabajo autónomo. (Inf4Ref26)

La metodología utilizada debe conducir a la participación e integración del alumnado implicándolo dialógicamente en la dinámica de clase.

Mi trabajo en el aula siempre suele ser muy participativo. Hoy de hecho un alumno me ha ayudado a explicar un problema. Yo estaba sentado en la mesa y el alumno estaba en la pizarra. Entre todos intentamos hacer el ejercicio de forma que todos interveníamos. Nunca he sabido cómo llamarle a esa técnica. Yo la llamo dialógica y la utilizo para integrar a todos en una dinámica participativa. (Inf1Ref29)

Es necesario “corregir sin criticar” (Inf.1Ref27), evitar que se sientan juzgados como personas. Pero ante todo reconocer el trabajo realizado, hacerles ver que van avanzando para motivarles y que descubran que son capaces. Todo trabajo debe ser corregido y devuelto al alumnado para que vean su progreso.

Les insisto mucho en que están aquí para aprender, y que se aprende equivocándose. ro saco un chaval a la pizarra y, quizás, tenga que acompañarlo paso a paso para que realice el ejercicio. Pero cuando termina le digo: 'muy bien'. Esta es la forma de reconocer los pequeños avances que van consiguiendo. A una alumna que nunca me hace nada pero de pronto empieza a traerme un trabajo le digo: 'muy bien, me encanta que me hayas hecho el resumen, me encanta que me hayas hecho los ejercicios'. Hacerles ver que lo que están haciendo sirve de algo, crearles expectativas de que pueden aprobar, porque pienso que es muy negativo el sentimiento de que no pueden; $y$ frente al mismo lucho. (Inf.1Ref27)

\title{
Contenidos
}

Dos principios caracterizan el desarrollo de los contenidos en el aula de prediversificación: flexibilización y centrarse en lo básico. La flexibilización implica ir a lo fundamental, haciendo hincapié, sin olvidar el currículum establecido, en aquellos elementos que conectan con su vida.

Lo que hago es ceñirme a lo que marcan los contenidos establecidos, pero siendo flexible. Unas veces hago hincapié en lo que les interesa más a ellos, otras, tengo que incidir en la importancia de aspectos que no les interesan, despertándoles el interés o incidiendo en la utilidad que tienen para sus vidas. Siempre pienso en su trayectoria, en sus puntos de interés para ir seleccionando contenidos, asegurando cubrir los básicos. (Inf4Ref24)

Esta flexibilización curricular reclama centrarse en el conjunto de aprendizajes o competencias clave que deben de asegurar la enseñanza obligatoria. El profesorado 
se refiere continuamente a los déficits en lectoescritura, a la falta de competencia comunicativa, como un elemento que determina la metodología y el desarrollo curricular en el aula. (Inf3Ref24)

En $4^{\circ}$ no doy tanta sintaxis porque es demasiado abstracto. Me interesa que conozcan las clases de palabras, para qué sirven... Pero sobre todo me interesa que sepan expresarse, que sepan escribir con corrección, que aprendan a utilizar registros distintos en cada situación comunicativa. (Inf4Ref18)

La evaluación

En este ámbito de individualización y flexibilización la evaluación ocupa un lugar central como vórtice que genera experiencias positivas y motivadoras en el alumnado al ver el resultado de su trabajo. El modelo de evaluación es formativa y continua, $e$ integra el trabajo en clase, el trabajo en casa y los exámenes que se realizan a lo largo del curso. (Inf1Ref34)

Mis alumnos lo saben: 'la evaluación no son los exámenes'. Los exámenes son un mecanismo de control, pero la evaluación es más global. Evaluamos todo el proceso. Diversifico los instrumentos para tener en cuenta la actitud, los trabajos de clase y de casa, y evidentemente, los exámenes. Todo hay que tenerlo en cuenta. (Inf4Ref29)

b) La experiencia del alumno

Esta medida para el alumnado constituye una experiencia de cambio.

Cuando se les atiende así ellos experimentan personalmente un gran cambio. Porque participan, trabajan, se sienten importantes, se ven capaces de aprobar y saben lo que es superar una dificultad. Es verdad que les ofrecemos siempre las medidas que les ayuden, pero para eso estamos y es nuestra labor; y no poner trabas o vallas altísimas para que sólo sean alcanzables para unos pocos. (Inf4Ref5)

El análisis de conglomerados pone en relación el conjunto de experiencias positivas de cambio, generadas en el alumnado, con los principios metodológicos anteriormente expuestos: "sentirse seguro e importante, redescubrirse como protagonista de su aprendizaje" (Inf4Ref5), "no sentirse distinto del resto de alumnos" (Inf3Ref14), "confiar en sus posibilidades al verse capaces de aprobar" (Inf4Ref5). Este conjunto de experiencias generan motivación y mejora de su autoestima al recorrer con éxito su trayectoria escolar.

Se han ido motivando en la medida que han ido viendo que eran capaces de hacer cada vez más cosas, se han ido animando. Estaban muy acostumbrados a suspender con resultados malísimos en cualquier prueba, cualquier control. Veían que el resto de la clase iba haciendo cosas que ellos no podían. $Y$ ahora al mejorar sus resultados escolares se van motivando, van introduciéndose en la rueda para ir superándose. (Inf3Ref1 1)

\section{Conclusiones y discusión}

El profesorado, definiendo y caracterizando la medida de apoyo que han puesto en marcha, enfatiza la idea de que las trayectorias de no éxito y creciente vulnerabilidad educativa se pueden cambiar/revertir. De este modo, la proponen como una medida preventiva y transitoria, en la que se cuidan no depreciar el valor de los aprendizajes imprescindibles y que garanticen el éxito para todos. Lo que es consistente con las diferentes propuestas que, desde la perspectiva frente a la exclusión, se realizan a la escuela para hacer posible una buena escuela para todos (Escudero, González González y Martínez Domínguez, 2009; Tarabini et al., 2015). 
El análisis de la voz del profesorado informante ha permitido también identificar los dos bloques temáticos principales que articula su discurso: de una parte, las personas y sus casuísticas (el alumnado); por otra, los espacios en los que se trabaja, grupo-clase de referencia y grupo de "prediversificación".

En torno al componente personal del alumnado, es clave tomar en consideración que la flexibilización (currículum, agrupamientos, metodología, tareas) ayuda a darle una respuesta y hacer posible su aprendizaje en condiciones equiparables en resultados con los del grupo referencia. Lo que es motivante para el alumnado y tiene impacto positivo en la minimización los efectos de vulnerabilidad personal y escolar. Afirman que están atrapados en un círculo vicioso de bajo rendimiento y desmotivación, que los hace seguir sacando malas notas y perder aún más su compromiso con su escuela. Por lo que restaurar la confianza y las expectativas de este alumnado es clave de éxito. Lo que es consistente con los estudios de Correa, Saldívar y López (2015) y de Longás y otros (2016). Para ello deben tener opciones de poder alcanzar los mismos aprendizajes que el resto de sus colegas del grupo referencia, llegándose a sentir capaces de aprender y aprobar (Valls y Kyriakides, 2013). Consideran vital que se den opciones de participación auténtica del alumnado en su proceso de aprendizaje y que se haga desde un currículum más democrático y una escuela alternativa (Feito, 2010).

En cuanto a los escenarios de interacción y aprendizaje, muestran una gran contraposición dual entre el grupo de referencia y el de grupo que trabaja de forma más personalizada y con un currículum más globalizado. Afirman que si se trabaja con grupos pequeños y con metodologías alternativas disminuye drásticamente el fracaso. Aspecto éste ya muy estudiado (Valls y Kyriakides, 2013; Valls, Prados y Aguilera, 2014), con resultados bastante coincidentes. El primero de ellos es considerado como más masificado, acelerado y poco atento a la diversidad y a las necesidades del alumnado, en el que predominan metodologías más tradicionales y academicistas. Mientras que el grupo que trabaja por ámbitos, al tener menos alumnado, permite una atención más personalizada y una flexibilización de los procesos y del currículum con metodologías alternativas. Ellos denuncian con contundencia que la clase tradicional no funciona y reproduce los factores de las escuelas ineficaces (Hernández-Castilla, Murillo y Martínez-Garrido, 2013). Al tiempo que describiendo su propuesta de medida de atención y los principios pedagógicos que la sustentan, lanzan una llamada de atención al resto de la escuela por si se está sobrecargando el currículum, adornándolo o dejando de lado lo esencial: el verdadero aprendizaje de todos.

Estas temáticas están permeadas por dos componentes clave: la idea de proceso y la constante permanencia de una filosofía pedagógica para la inclusión. En este sentido, en contraste con los resultados obtenidos por Tarabini (2015), el profesorado informante se siente concernido con las condiciones y causas que van consolidando las trayectorias de fracaso y asumen actuar frente a las mismas. Lo que se traduce en entrar a debate en dimensiones clave de la atención a este colectivo de chicos y que actúan de forma preventiva o como motor de exclusión, como son contenidos, procesos de enseñanzaaprendizaje, metodología, evaluación y rol del alumnado en el aula.

Un tema estrella para este profesorado es que para atender a la diversidad hay que ir a aprendizajes más profundos y estables, insistiendo en tareas que les lleven a las competencias básicas y los aprendizajes imprescindibles. Son varios los trabajos que insisten en esta línea (Bolívar, 2008; Coll, 2006). En este sentido, afirman que las 
medidas de atención a la diversidad deben compartir objetivos y contenidos con el aula ordinaria, mientras que lo que se cambie sea principalmente la metodología de trabajo y la ratio. Lo que es consistente con otras investigaciones similares (Ainscow, Booth y Dyson, 2006; Escudero, 2005, 2013).

Críticos con la normal escolarización en secundaria, proponen esta medida precoz para prevenir la profundización de las trayectorias de fracaso y exclusión del éxito de estos colectivos. Pero conscientes de que ello puede ayudar a la dualización del sistema (Canario, 2000; Correia, 2004), señalan que, lejos de ser una medida paliativa -para trayectorias consumadas de fracaso-, proponen medidas blandas que intervienen en necesidades particulares y puntuales, dando opción a actuar preventivamente sin que marque trayectorias (Escudero, 2005, 2013), y no dejando actuar otras inercias que silencian, ocultan o terminan justificando los resultados (Karsz, 2004). Con todo ello, infieren un conjunto de reflexiones para una escuela secundaria inclusiva que minimice los factores que llevan al fracaso y al abandono temprano. Todas ellas bastante consistentes con las propuestas de Luzón y sus colaboradores (2009), la OCDE (2016) o Sammons y Bakkum (2011).

En definitiva, a la luz de sus declaraciones, el profesorado implicado en el desarrollo de estas medidas de apoyo, se han ido formando una conciencia pedagógica, que camina hacia una identidad inclusiva y beligerante con la apatía y la desesperanza de pensar que ante el tema del fracaso nada se puede hacer. Aspecto que demandaba Zeichner (2010) al hablar de una nueva formación del profesorado. Este profesorado es el más implicado en atención a la diversidad (llevan estas medidas y altas capacidades), así como con mayor experiencia y participación en el grupo motor del centro (Díaz-Gibson, Civís, Carrillo y Cortada, 2015). Lo que es tanto una limitación del estudio, como una muestra de que parte de la eficacia de este centro escolar está en saber equilibrar calidad y equidad, promoviendo un proyecto, medidas y estructuras que coadyuven a alcanzar el éxito para todos (Ainscow y West, 2008; Murillo y Duk, 2011), y, entre ellas, derivar precozmente al profesorado más consciente hacia los colectivos en riesgo para garantizar la equidad y facilitar su éxito, aunque obvia importantes acciones de cara a mejorar la colaboración entre el profesorado y la transferencia al aula ordinaria (Gómez, Petreñas, Sabando y Puigdellívol, 2017), que sí deberían tomarse en consideración.

Lo que se aprende de atender bien a la diversidad (en grupos flexibles y que trabajan por ámbitos, en este caso) puede ayudar también al resto de la escuela y debiese ser tomado en consideración, aunque obviamente eso suponga cambios y adecuaciones. Y eso hay que asumirlo. González advierte en este sentido que "cualquier centro escolar que se plantee mejorar con vistas a enganchar más a sus alumnos y evitar su abandono difícilmente podrá llegar a ello si no replantea y re-considera [globalmente] tanto sus facetas más pedagógicas como organizativas" (2015, p. 173).

\section{Agradecimientos}

$\mathrm{Al}$ profesorado y equipo directivo del Instituto de Educación Secundaria "El Palmeral" de Vera (Almería), quien abrió sus puertas y ofreció su conocimiento práctico profesional a debate.

\section{Referencias}


Ainscow, M. y West, M. (2008). Mejorar las escuelas urbanas: Liderazgo y colaboración. Madrid: Narcea.

Ainscow, M., Booth, T. y Dyson, A. (2006). Improving schools, developing inclusion. Londres: Routledge.

Aramendi, P., Vega, A. y Santiago, K. (2011). Los programas de atención a la diversidad en la Educación Secundaria desde la perspectiva de los estudiantes: Estudio comparado. Revista de Educación, 356, 185-209.

Armstrong, A. C., Armstrong, D. y Spandagou, I. (2009). Inclusive education: International policy \& practice. Londres: Sage.

Birks, M. y Mills, J. (2015). Grounded theory: A practical guide. Londres: SAGE.

Bolívar, A. (2005). Equidad educativa y teorías de la justicia. REICE. Revista Iberoamericana sobre Calidad, Eficacia y Cambio en Educación, 3(2), 42-69.

Bolívar, A. (2008). Ciudadanía y competencias básicas. Sevilla: Fundación ECOEM.

Canario, R. (2000). Territorios educativos de intervenção prioritaria: A escola face a exclução Social. Revista de Educação, 9(1), 125-134.

Clandinin, D. J. y Connelly, F. M. (2000). Narrative inquirity: Experience and story in qualitative research. Nueva York, NY: Teachers College Press.

Coll, C. (2006). Lo básico en la educación básica. Reflexiones en torno a la revisión y actualización del currículo de la educación básica. Revista Electrónica de Investigación Educativa, 8(1), 117.

Correa, F. E., Saldívar, A. y López, A. D. (2015). Autoconcepto y estados emocionales: Su relación con la motivación en adolescentes. Enseñanza e Investigación en Psicología, 20(2), 173-183.

Correia, J. A. (2004). A construção político-cognitiva da exclusão social no campo educativo. Revista Educação Unisinos, 5(9), 217-246.

Darling-Hammond, L. (2001). El derecho de aprender. Crear buenas escuelas para todos. Barcelona: Ariel.

Díaz-Gibson, J., Civís, M., Carrillo, M. y Cortada, E. (2015). El liderazgo y la gobernanza colaborativa en proyectos educativos comunitarios. Pedagogía Social. Revista Interuniversitaria, 26, 59-83. doi:10.7179/PSRI_2015.26.03

Echeita, G., Simón, C., Verdugo, M. A., Sandoval, M., López, M., Calvo, I. y González, F. (2009). Paradojas y dilemas en el proceso de inclusión educativa en España. Revista de Educación, 349, 153-178.

Escudero, J. M. (2005). Fracaso escolar, exclusión educativa ¿De qué se excluye y cómo? Profesorado, Revista de Currículum y Formación del Profesorado, 9(1), 1-24.

Escudero, J. M. (Coord.). (2013). Estudiantes en riesgo, centros escolares en riesgo: Respuestas educativas al alumnado en situaciones de vulnerabilidad. Murcia: DM.

Escudero, J. M. y Rodríguez, M. J. (2016). Riesgo, fracaso y abandono escolar temprano: Mejorar la comprensión, las políticas y las prácticas. En J. M. Escudero (Comp.), Inclusión y exclusión educativa: Realidades, miradas y propuestas (pp. 77-114). Valencia: Nau Llibres.

Escudero, J. M., González González, M. T. y Martínez Domínguez, B. (2009). El fracaso escolar como exclusión educativa: Comprensión, políticas y prácticas. Revista Iberoamericana de Educación, 50, 41-64.

Feito, R. (2010). De las competencias básicas al currículum integrado. Qurriculum, 23, 55-79. 
Fernández, M., Mena, L. y Reviere, J. (2010) Fracaso y abandono escolar en España. Barcelona: Fundación la Caixa.

Gómez, J., Latorre, A., Sánchez, M. y Flecha, R. (2006). Metodología comunicativa crítica. Barcelona: El Roure.

Gómez, G., Petreñas, C., Sabando, D. y Puigdellívol, I. (2017). The role of the Support and Attention to Diversity Teacher (SADT) from a community-based perspective: Promoting educational success and educational inclusion for all. Teaching and Teacher Education, 64, 127-138. doi:10.1016/j.tate.2017.02.002

González González, M. T. (2015). Los centros escolares y su contribución a paliar el desenganche y abandono escolar. Profesorado, Revista de Currículum y Formación del Profesorado, 19(3), 158-176.

Hernández-Castilla, R., Murillo, F. J. y Martínez-Garrido, C. (2013). Factores de ineficacia escolar. REICE. Revista Iberoamericana sobre Calidad, Eficacia y Cambio en Educación, 12(1), 103-118.

Jiménez, M., Luengo, J. y Táberner, J. (2009). Exclusión social y exclusión educativa como fracasos. Conceptos y líneas para su comprensión e investigación. Profesorado, Revista de Currículum y Formación del Profesorado, 13(3), 11-49.

Karsz, S. (Coord.). (2004). La exclusión: Bordeando sus fronteras. Definiciones y matices. Barcelona: Gedisa.

Longás, J., Cussó, I., De Querol, R. y Riera, J. (2016). Análisis de factores de apoyo a trayectorias de éxito escolar en la enseñanza secundaria en contextos de pobreza y vulnerabilidad social en España. Un estudio de casos múltiples. REXE-Revista de Estudios y Experiencias en Educación, 15(28), 107-127. doi:10.21703/rexe.2016281071276

Luzón, A., Porto, M, Torres, M. y Ritacco, M. (2009). Buenas prácticas en los programas extraordinarios de atención a la diversidad en centros de educación secundaria. Una mirada desde la experiencia. Profesorado, Revista de Currículum y Formación del Profesorado, 13(3), 216-238.

Martínez, B. (2010). Claves para repensar la organización de una escuela inclusiva que evite la dualización interna de la enseñanza. En A. Manzanares (Ed.), Organizar y dirigir en la complejidad. Instituciones educativas en evolución (pp. 379-389). Madrid: Wolters Kluwer Educación.

Murillo, F. J. (2004). La investigación sobre eficacia escolar a debate. Análisis de las críticas y aportaciones. Tendencias Pedagógicas, 9, 111-131.

Murillo, F. J. y Duk, C. (2011). ¿Escuelas eficaces versus inclusivas? Revista Latinoamericana de Educación Inclusiva, 5(1), 11-12.

Murillo, F. J. y Hernández-Castilla, R. (2011). La equidad en la investigación sobre eficacia escolar. Profesorado, Revista de Currículum y Formación del Profesorado, 15(3), 3-8.

OCDE. (2016). Estudiantes de bajo rendimiento. Por qué se quedan atrás y cómo ayudarles a tener éxito. Recuperado de http://www.oecd.org/pisa/keyfindings/PISA-2012-Estudiantes-de-bajorendimiento.pdf

OCDE, OIE-UNESCO y UNICEF LACRO. (2016). La naturaleza del aprendizaje: Usando la investigación para inspirar la práctica. Recuperado de http://www.unicef.org/lac/20160505_UNICEF_UNESCO_OECD_Naturaleza_Aprendi zaje_.pdf 
Orden de 25 de julio de 2008 , por la que se regula la atención a la diversidad del alumnado que cursa la educación básica en los centros docentes públicos de Andalucía. Boletín Oficial de la Junta de Andalucía, $\mathrm{n}^{\circ}$ 167, de 22 de agosto de 2008. Recuperado de http://www.juntadeandalucia.es/boja/2008/167/2

Parrilla, A. (2009). ¿ YY si la investigación sobre inclusión no fuera inclusiva? Reflexiones desde una investigación biográfico-narrativa. Revista de Educación, 349, 101-118.

Rodríguez, A. M. (2013). Modelos de atención a la diversidad en educación secundaria obligatoria: Análisis comparativo de los planes de atención a la diversidad de las comunidades autónomas de Andalucía y de la Región de Murcia. Revista Nacional e Internacional de Educación Inclusiva, 6(3), 41-64.

Sammons, P. y Bakkum, L. (2011). Effective schools, equity and teacher efficacy: A review of the literature. Profesorado, Revista de Currículum y Formación del Profesorado, 15(3), 9-26.

Sammons, P. (2007). School effectiveness and equity: Making connections. Reading: Centre for British Teachers.

Sevilla, D. (2003). La educación comprensiva en España: Paradoja, retórica y limitaciones. Revista de Educación, 330, 35-57.

Stake, R. E. (2007). Investigación con estudio de casos. Madrid: Morata.

Susinos, T. y Parrilla, A. (2008). Dar la voz en la investigación inclusiva. Debates sobre inclusión y exclusión desde un enfoque biográfico-narrativo. REICE. Revista Iberoamericana sobre Calidad, Eficacia y Cambio en Educación, 2(6), 157-71.

Tarabini, A. (2015). Políticas de lucha contra el abandono escolar en España. Madrid: Síntesis

Tarabini, A., Curran, M., Montes, A. y Parcerisa, L. (2015). El rol de los centros educativos en la prevención del abandono escolar: Una aproximación desde la perspectiva micropolítica. Educação, Sociedade छ Culturas, 45, 121-141.

UNESCO. (2008). Educación de calidad, equidad y desarrollo sostenible: Una concepción holística inspirada en las cuatro conferencias mundiales sobre la educación que organiza la UNESCO en 2008-2009. Recuperado de http://www.unesco.org/education/Synergies4conferences.pdf

Valls, R. y Kyriakides, L. (2013). The power of interactive groups: How diversity of adults volunteering in classroom groups can promote inclusion and success for children of vulnerable minority ethnic populations. Cambridge Journal of Education, 43(1), 17-33. doi:10.1080/0305764X.2012.749213

Valls, R., Prados, M. M. y Aguilera, A. (2014). El proyecto INCLUD-ED: Estrategias para la inclusión y la cohesión social en Europa desde la educación. Investigación en la Escuela, 82, 31-343.

Van Dijk, T. A. (2012). Discurso y contexto. Un enfoque sociocognitivo. Barcelona: Gedisa.

Vega, A. y Aramendi, P. (2009). La atención a la diversidad: Interrogantes para la iniciación profesional de los «fracasados». Enseñanza छ Teaching: Revista Interuniversitaria de Didáctica, 27(1), 157-170.

Zeichner, K. M. (2010). La formación del profesorado y la lucha por la justicia social. Madrid: Morata.

\section{Breve CV de los autores}

\section{Jesús Domingo}


Catedrático de la Universidad de Granada, especialista en formación, asesoría y liderazgo para la inclusión de colectivos vulnerables, la mejora de la educación pública y la justicia social. Es miembro del grupo de investigación FORCE, de la Red de Investigación RILME y del Proyecto Atlántida. IP y participante en una veintena de proyectos de investigación y de cooperación internacional financiados con fondos públicos y autor de más de un centenar de publicaciones en esta línea de investigación. ORCID ID: 0000-0002-8319-5127. Email: jdomingo@ugr.es

\section{José Manuel Martos}

Doctor en Ciencias de la Educación, miembro del grupo de investigación FORCE de la Universidad de Granada y del Proyecto Atlántida. Sus líneas de investigación son la desigualdad educativa y el análisis discursivo en redes sociales. Trabaja desde la metodología biográfico-narrativa, el análisis crítico del discurso y la teoría fundamentada. ORCID ID: 0000-0001-5501-3077. Email: jmmartos@ugr.es 